\title{
Experimental demonstration of a magnifying prism hyperlens at $\mathrm{THz}$ frequencies
}

Habib, Md Samiul; Stefani, Alessio; Atakaramians, Shaghik; Fleming, Simon C.; Argyros, Alexander; Kuhlmey, Boris T.

Published in:

Proceedings of 11 th International Congress on Engineered Material Platforms for Novel Wave Phenomena

Link to article, DOI:

10.1109/MetaMaterials.2017.8107865

Publication date:

2017

Document Version

Peer reviewed version

Link back to DTU Orbit

Citation (APA):

Habib, M. S., Stefani, A., Atakaramians, S., Fleming, S. C., Argyros, A., \& Kuhlmey, B. T. (2017). Experimental demonstration of a magnifying prism hyperlens at THz frequencies. In Proceedings of 11th International Congress on Engineered Material Platforms for Novel Wave Phenomena (pp. 133-135). [8107865] IEEE. https://doi.org/10.1109/MetaMaterials.2017.8107865

\section{General rights}

Copyright and moral rights for the publications made accessible in the public portal are retained by the authors and/or other copyright owners and it is a condition of accessing publications that users recognise and abide by the legal requirements associated with these rights.

- Users may download and print one copy of any publication from the public portal for the purpose of private study or research.

- You may not further distribute the material or use it for any profit-making activity or commercial gain

- You may freely distribute the URL identifying the publication in the public portal 


\title{
Experimental demonstration of a magnifying prism hyperlens at $\mathrm{THz}$ frequencies
}

\author{
Md. Samiul Habib ${ }^{1}$, Alessio Stefani ${ }^{1,2}$, Shaghik Atakaramians ${ }^{1}$, Simon C. Fleming ${ }^{1}$, Alexander Argyros ${ }^{1}$, \\ and Boris T. Kuhlmey ${ }^{1,3}$ \\ ${ }^{1}$ Institute of Photonics and Optical Science (IPOS), School of Physics, The University of Sydney, Sydney, \\ New South Wales, 2006, Australia \\ ${ }^{2}$ DTU Fotonik, Department of Photonics Engineering, Technical University of Denmark, DK-2800 Kgs. \\ Lyngby, Denmark \\ ${ }^{3}$ Centre for Ultrahigh Bandwidth Devices for Optical Systems (CUDOS), School of Physics, The \\ University of Sydney, Sydney, New South Wales, 2006, Australia \\ e-mail : samiul.habib@sydney.edu.au
}

\begin{abstract}
We experimentally demonstrate a magnifying wire medium (WM) prism hyperlens at $\mathrm{THz}$ frequencies. The different lengths of wire in the prism have different resonance frequencies, so that there is no frequency at which a good image is possible. We show that using spatially varying time gating or frequency convolution the resonant response can be removed and experimentally demonstrate sub-diffraction magnified imaging of a sub-wavelength double aperture.
\end{abstract}

\section{INTRODUCTION}

The diffraction limit is a fundamental restriction of any conventional imaging systems, preventing sub-wavelength details to be transmitted in the far-field. To overcome the diffraction limit, hyperbolic metamaterials can be used since they have large anisotropy leading to a hyperbolic dispersion relation [1] and support propagating extraordinary waves containing high spatial frequencies. At low frequencies, in the canalization regime, all spatial frequencies propagate with identical phase velocity, so that images propagate without diffraction or distortion over many wavelengths [2]. To date, in order to magnify images to the far-field, different designs of hyperlenses have been reported [3-4], including sub-wavelength alternating layers of metal and dielectric in a cylindrical geometry. At THz and lower frequencies hyperlenses can be made using WM which have low loss [5]. In 2013 Tuniz et al., experimentally demonstrated both magnifying and non-magnifying hyperlens using the fiber drawing technique [6]. The magnifying hyperlens was made using a tapered section of WM, however, fabrication of such large tapers is a challenging issue.

Most previous designs for image magnification rely on either curved geometry or tapering of the medium, which can be difficult to fabricate. Here, we experimentally demonstrate a prism design of magnifying hyperlens analogous to an early design for magnifying hyperlens based on layered media [3]. The main advantage of the prism geometry is that it does not rely on curved geometry or tapering, making the fabrication relatively simpler at low frequencies.

In this paper, we experimentally demonstrate transmission of near-field information through the hyperlens, however, our experiments show the existence of imaging artefacts due to reflections supported by the WM. In order to eliminate the reflections, we use position dependent convolution so that broad-band imaging is achieved.

\section{RESUlTS AND DISCUSSIONS}

Figure 1(a) shows a schematic of the magnifying WM prism hyperlens. The wires within the hyperlens are directed along z-axis and we measure the fields near to the elongated side of the hyperlens. The length of the longest wire is $L_{l}=3 \mathrm{~mm}$, that of the shortest wire is $L_{s}=1.56 \mathrm{~mm}$ and the length difference between consecutive wires is $90 \mu \mathrm{m}$. Such a geometry will provide a two-fold magnified image at the output. The WM presented here contains 443 indium wires, arranged in hexagonal lattice, with an average $10 \mu \mathrm{m}$ diameter and $50 \mu \mathrm{m}$ pitch at 


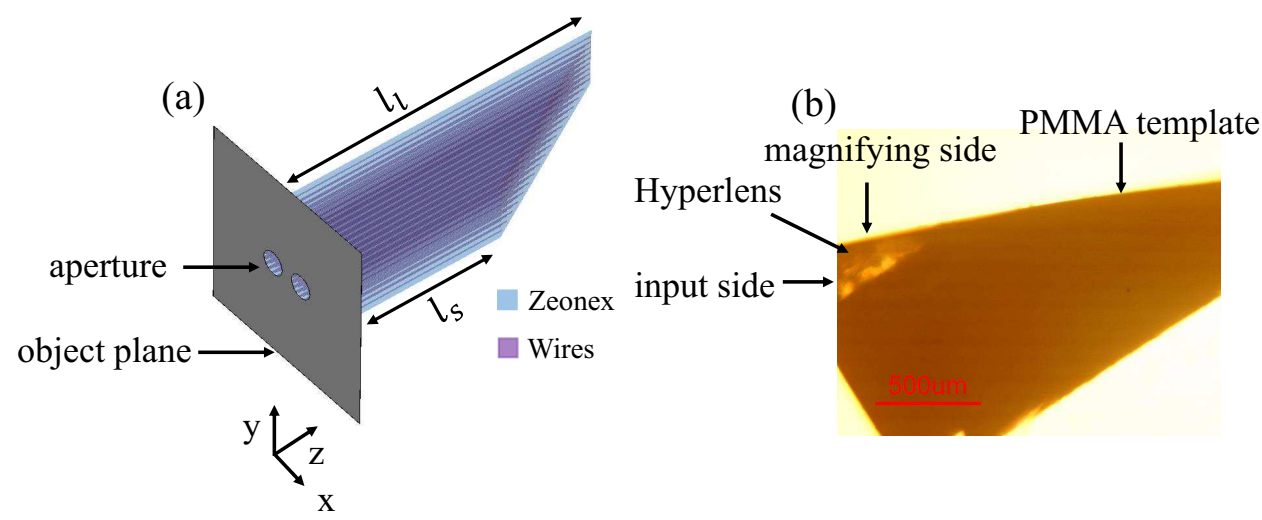

Fig. 1: (a) Schematic of the prism design of magnifying hyperlens. The diameter of the apertures is $200 \mu \mathrm{m}$ with inner edge-to-edge separation of $100 \mu \mathrm{m}$. (b) Microscopy image of the side view of the fabricated hyperlens.

the fiber end, hosted in the Zeonex. As a dielectric Zeonex is chosen because it has relatively low loss at $\mathrm{THz}$ frequencies. We use an inexpensive technique to produce the final shape of the hyperlens. For this, we take a PMMA template of thickness $\sim 2 \mathrm{~mm}$, and then cut this template with the required angle. After that, we drill a hole $\sim 1.5 \mathrm{~mm}$ into the template, insert the fiber into it. Figure 1(b) shows microscopy image of side view of the fabricated hyperlens. Finally, to get good flatness at both ends of the fiber, we polish both sides using regular polishing paper. We characterize the transmission properties of the hyperlens via time resolved $\mathrm{THz}$ time domain spectroscopy [7]. In the experiment, two sub-wavelength apertures are placed in direct contact with the input side of the hyperlens. The size of the apertures are $200 \mu \mathrm{m}$ with inner edge-to-edge separation of $100 \mu \mathrm{m}$, such dimensions are below the diffraction limit over the frequency range considered, i.e., $0.2-0.6 \mathrm{THz}$. $x$-polarized $\mathrm{THz}$ pulses are used to excite the apertures, while near-field information is measured at the elongated side of the hyperlens using near-field detector antenna. Figure 2(a) shows the experimental frequency dependent near-field intensity profile, which is normalized with the input pulse spectrum.
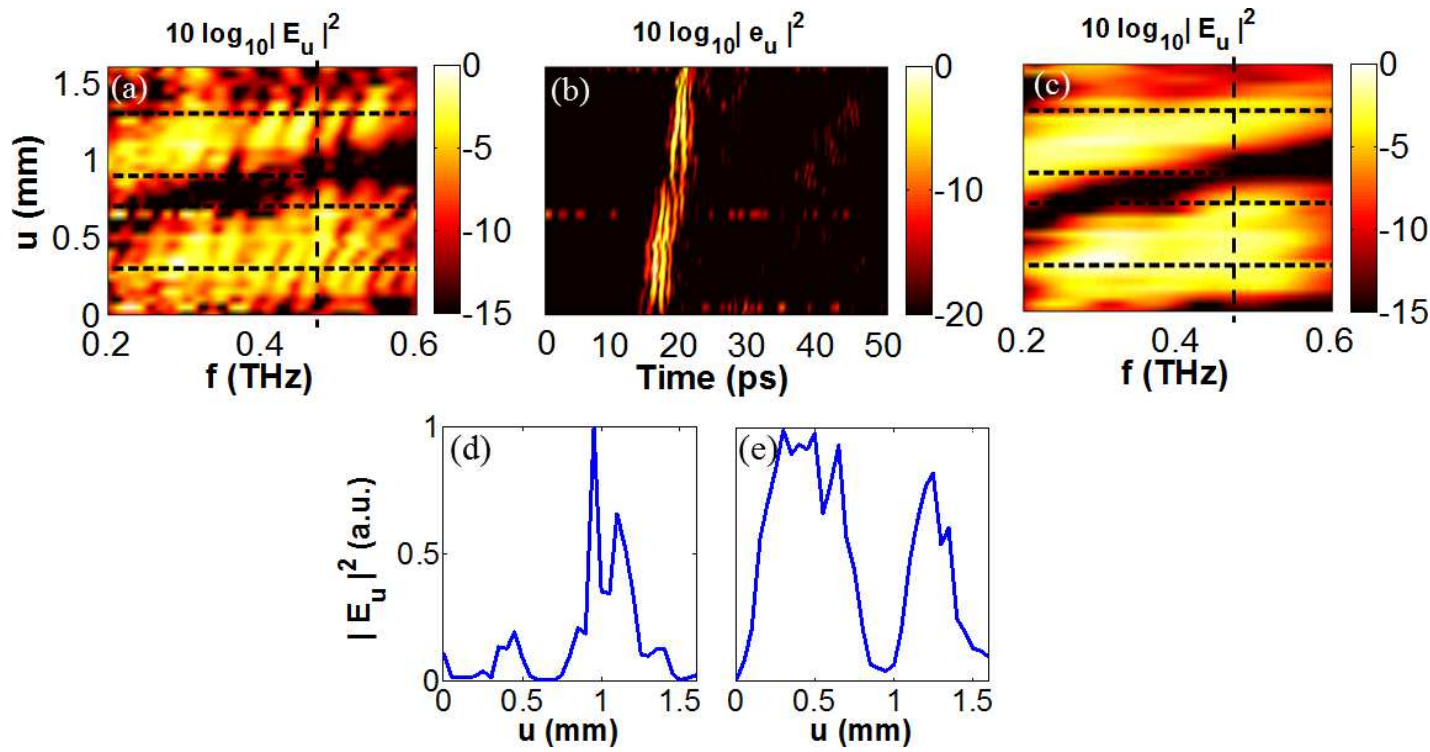

Fig. 2: (a,b) Experimental frequency dependent profile and temporal intensity profile along the $u$-axis through the middle of the hyperlens. (c) Experimental intensity profile after applying the space dependent convolution. Intensity $(y=0)$ at $0.47 \mathrm{THz}(\mathrm{d})$ before convolution and (e) after convolution. 
From the intensity profile, we see two intensity maxima locating the apertures positions, indicating that our device is capable of separating feature sizes below the diffraction limit. The horizontal dotted lines in Fig. 2(c) indicates location of the imaged apertures, providing a two-fold magnified image. In spite of the strong field concentration at the apertures position, we also see deterioration of the image at some frequencies. This deterioration of image is due to the resonances of the individual wires. Since the fields take different time to reach the output interface, the temporal intensity profile appears slanted. From the temporal profile the main pulse and first reflections can be seen, whereas the intensity of the first reflection is very weak, and is separated by $16.0 \pm 0.5 \mathrm{ps}$ to the main pulse. We observe that such low intensity reflections can degrade the image quality, see the vertical dotted line in Fig. 2(a).

It is known that, for straight uniform WM, high quality images can be obtained at the Fabry-Perot resonances (FPRs) of the wires, however, away from such resonances images deteriorate [6]. Kaltenecker et al., experimentally demonstrated that such image deterioration is due to the reflections supported by the WM, and is possible to eliminate the subsequent reflections by appropriate time-gating [8]. However, for prism geometry where wires are of varying lengths, this results in different resonance frequencies of individual wires. As a consequence, there is no frequency at which imaging is good. In that particular geometry, time gating of ultra-short pulses needs to be adjusted according to the varying length of each wire. In the frequency domain such time-gating can be approximated by space dependent convolution [7]. Figure 2(c) shows frequency dependent intensity profile after applying the position dependent convolution method. The intensity (at $y=0$ ) at $0.47 \mathrm{THz}$ is plotted in Fig. 2(d), which is difficult to interpret, and after applying the convolution method two apertures can be clearly separated, Fig. 2(e), leading to a significant improvement in imaging.

\section{CONCLUSION}

In conclusion, a magnifying wire medium prism hyperlens is presented which does not rely on tapering for image magnification, and is easier to fabricate. We have experimentally demonstrated that the hyperlens can separate two sub-wavelength apertures, providing a two-fold magnified image at the output. Magnification of the hyperlens can be scaled up by properly adjusting the prism angle. However, imaging from such hyperlenses is not perfect: reflections supported by the WM distort the image quality. For this, we have adapted a space dependent filtering technique equivalent to spatially variable time gating and demonstrated it yields so broad-band imaging.

\section{ACKNOWLEDGEMENT}

This research was supported by the Australian Research Council (ARC) under the Discovery Project scheme number DP120103942, and partly under the ARC Centre of Excellence scheme CUDOS (CE110001018). A.S. acknowledges the Eugen Lommel Stipend and Marie Sklodowska-Curie grant of the European Unions Horizon 2020 research and innovation programme (708860). S.A. acknowledges a support of ARC funding DE140100614.

\section{REFERENCES}

[1] A. Poddubny, I. Iorsh, P. Belov, and Y. Kivshar, "Hyperbolic metamaterials," Nat. Photon., vol. 7, p. 948-957, 2013.

[2] C. R. Simovski, P. A. Belov, A. V. Atrashchenko, and Y. S. Kivshar, "Wire Metamaterials: Physics and Applications," Adv. Mater., vol. 24, p. 4229-4248, 2012.

[3] A. Salandrino and N. Engheta, "Far-field subdiffraction optical microscopy using metamaterial crystals: Theory and simulations," Phys. Rev B, vol. 74, p. 075103, 2006.

[4] Z. Jacob, L. V. Alekseyev, and E. Narimanov, "Optical Hyperlens: Far-field imaging beyond the diffraction limit," Opt. Express, vol. 14, p. 8247-8256, 2006.

[5] P. A. Belov, Y. Hao, and S. Sudhakaran, "Subwavelength microwave imaging using an array of parallel conducting wires as a lens," Phys. Rev B, vol. 73, p. 033108, 2006.

[6] A. Tuniz, K. J. Kaltenecker, B. M. Fischer, M. Walther, S. C. Fleming, A. Argyros, and B. T. Kuhlmey, "Metamaterial fibres for subdiffraction imaging and focusing at terahertz frequencies over optically long distances," Nat. Commun., vol. 4, p. 2706, 2013.

[7] M. S Habib, A. Stefani, S. Atakaramians, S. C. Fleming, A. Argyros, and B. T. Kuhlmey, "A prism based magnifying hyperlens with broad-band imaging," Appl. Phys. Lett., vol. 110, p. 101106, 2017.

[8] K. J. Kaltenecker, A. Tuniz, S. C. Fleming, A. Argyros, B. T. Kuhlmey, M. Walther, and B. M. Fischer, "Ultrabroadband perfect imaging in terahertz wire media using single-cycle pulses," Optica, vol. 3, p. 458-464, 2016. 